\title{
SYNTAGMES NOMINAUX ET SYNTAGMES ADPOSITIONNELS DANS TROIS LANGUES EN CONTACT EN COTE D'IVOIRE
}

\author{
Béatrice Akissi Boutin \\ Université de Toulouse-Le Mirail \& Institut de Linguistique Appliquée \\ d'Abidjan
}

\begin{abstract}
Résumé
Le français distingue, à l'instar de nombreuses langues telles le dioula qui possèdent des adpositions, les syntagmes nominaux des syntagmes prépositionnels. Seuls les syntagmes nominaux peuvent assumer la fonction de sujet d'un verbe, alors que les deux types de syntagmes peuvent se trouver en fonction de complément d'un verbe ; c'est alors le verbe qui sélectionne le type de complément, nominal ou adpositionnel. Dans d'autres langues, au contraire, telles le baoulé, les éléments qui introduisent un syntagme nominal pour former un syntagme de rang supérieur ne constituent pas une classe d'adpositions. Le contact de ces deux langues en Côte d'Ivoire peut-il expliquer que le français local ivoirien donne à des syntagmes prépositionnels (de forme PREP (N/Pro) la faculté d'occuper des fonctions de syntagmes nominaux dans une phrase ? Notre discussion porte tout d'abord sur le statut des syntagmes adpositionnels en français, en dioula et en baoulé. Nous montrons que certaines de ces formes peuvent être analysées comme des syntagmes nominaux à tête vide. D’autres faits de langue sont ensuite rapprochés de ce phénomène.
\end{abstract}

\section{Introduction}

Il existe en français local ivoirien des usages particuliers de pour dans lesquels celui-ci, avec son complément, est apte à occuper des positions structurelles nominales ${ }^{1}$. Nous avons, par exemple :

1 Je remercie Jérémie Kouadio N'Guessan, Kouakou Kouamé et Kalilou Téra pour leur aide dans l'approfondissement du fonctionnement du baoulé et du dioula. Ma réflexion sur pour (N/Pro) a débuté

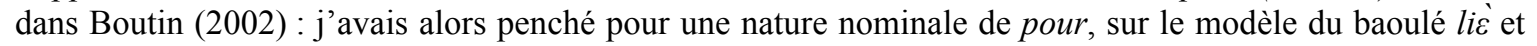
conclu sur une juxtaposition de pour avec son complément de nom. Cette solution n'était pas satisfaisante parce que peu cohérente avec d'autres faits du français de Côte d'Ivoire. La Journée d'Hommage à Suzanne Lafage, le 28/02/05 à la Maison des Sciences de l'Homme de Paris, m'avait donné l'occasion d'approfondir les faits selon une problématique double : déterminer le statut de pour et apporter l'éclairage des langues ivoiriennes en ce qu'elles peuvent interférer au niveau des représentations syntaxiques (Boutin 2006). La réflexion s'est poursuivie ensuite sur les locatifs (Boutin 2005), ce qui me permet aujourd'hui de balayer des faits plus larges et de les aborder avec une nouvelle problématique. 
(1) Avant de te moquer du linge de ta voisine, regarde si pour toi est propre (nouchi.com 2/13/2001) (Avant de te moquer du linge de ta voisine, regarde si le tien est propre) ${ }^{2}$

(2) Ce qui a fait dire à beaucoup que « couvre-feu de Gbagbo-là, ce n'est pas comme pour Guéï-là ! » (Soir Info 13.12.00)

(Ce qui a fait dire à beaucoup que le couvre-feu de Gbagbo n'est pas comme celui de Guéi)

(3) Le cours [de cuisine] sera le lundi à 16 heures 30 sauf pour le dernier lundi qui sera à 9 heures 30 (affiche en établissement technique)

(Le cours sera le lundi à 16 heures 30 sauf celui du dernier lundi qui sera à 9 heures 30)

Nous formulons l'hypothèse qu'il existe, dans cette variété de français, des syntagmes à tête vide, de structure formelle PREP ( $/$ Pro), qui ont un statut syntaxique de constituant nominal. En effet, ces exemples (1) à (3) sont à rapprocher d'autres constructions où des syntagmes prépositionnels sont en position de constituants nominaux, tels que (4) à (6). Nous donnons entre crochets le contexte situationnel des énoncés :

(4) Ton grand père [Le père de ton papa] est toujours là ?

- Non, il est décédé, c'est chez ma maman qui est encore là (oral 04)

(5) [Les clignotants] A droite s'allume mais à gauche reste éteint (oral 04)

(6) [Les bananes] C'est ici qui est mûr, dans la cour n'est pas mûr (oral 98)

(Ce sont celles d'ici qui sont mûres, celles dans la cour ne sont pas mûres [ou celles de la cour ne sont pas mûres])

Dans les formes PREP ( $N$ / Pro) telles qu'elles apparaissent dans (1) à (6), le référent du nom tête est implicite mais c'est bien lui, et non le nom complément de la préposition, qui est argument de la phrase. Si l'utilisation des formes PREP (N/Pro) semble atypique et que celles-ci ne sont attestées à ce jour dans aucune zone francophone hormis la Côte d'Ivoire où le français est en contact avec diverses langues, l'occasion d'examiner la possibilité d'une interférence est idéale. Nous entendons par interférence, à la suite de Weinreich (1953:1), le réarrangement d'un système qui résulte de l'introduction d'éléments d'autres systèmes à l'intérieur des domaines les plus hautement structurés de la langue. Dans le domaine syntaxique, ces éléments peuvent être abstraits et concerner des propriétés que certains morphèmes d'une langue acquièrent, sur le modèle des propriétés que leurs homologues, ou des morphèmes parallèles, ont dans les langues en contact. La première question qui se pose est donc de savoir si ces formes PREP ( $/$ Pro) peuvent être rapprochées des formes ( $N$ / Pro) POSTP des phrases suivantes :

$\begin{array}{llllll}\text { (7) dioula : } & \grave{a} & m \grave{a} & \grave{a} & \text { tá } & \text { móbili bòri, } \\ & \text { 3SG } & \text { PRF;NEG } & \text { 3SG } & \text { POSTP } & \text { voiture conduire, } \\ & \grave{a} & k \grave{a} & \grave{n} & \text { tá } & \text { bòri } \\ & \text { 3SG } & \text { PRF;POS } & 1 \mathrm{SG} & \text { POSTP } & \text { conduire }\end{array}$

(Il n'a pas conduit sa voiture, il a conduit la mienne)

${ }^{2}$ La traduction proposée n'est chaque fois qu'un équivalent sémantique possible, qui ne correspond pas toujours à un équivalent syntaxique. 


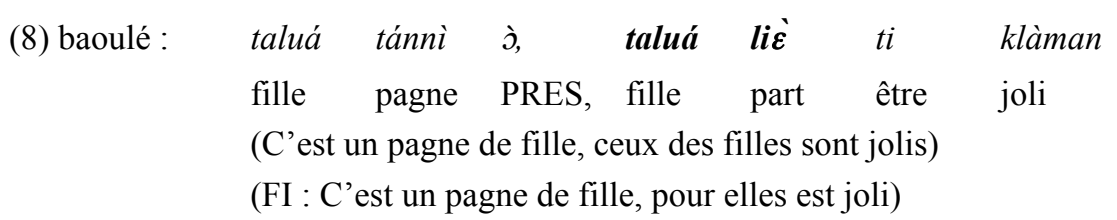

L'utilisation de structures adpositionnelles pour des fonctions nominales remettent-elles en cause la démarche de catégorisation en syntagmes nominaux et syntagmes adpositionnels ${ }^{3}$ ? La question peut-elle être traitée parallèlement dans les trois langues ? Autrement-dit, existe-t-il une définition commune de la classe des adpositions et, au-delà, de celles des syntagmes adpositionnels et des syntagmes adverbiaux dans les trois langues en contact? De fait, une description différentielle est nécessaire avant que l'on puisse évaluer dans quelle mesure le contact avec les langues ivoiriennes peut expliquer les particularités du français de Côte d'Ivoire étudiées ici. Les deux principaux facteurs de probabilité de transfert de fonctions grammaticales pour Weinreich seront discutés tout au long de l'étude: "The transferability of morphemes is considered as a correlate of their grammatical function in the source language and the resistance of the recipient language " (Weinreich $1953: 31$ ).

Les propriétés sémantico-syntaxiques et la structure interne des formes PREP (N / Pro) seront examinées conjointement aux formes ( $N /$ Pro) POSTP. Ainsi, après avoir vérifié la capacité des syntagmes adpositionnels en question à occuper des fonctions nominales (section 2), nous analysons, en français et dans les langues ivoiriennes en contact, l'existence de syntagmes nominaux à tête vide (section 3), les conditions pour que des syntagmes adpositionnels aient un statut nominal (section 4), les possibilités que des adverbiaux occupent des positions nominales (section 5).

Quatre langues ou variétés sont convoquées : le français standard, le français de Côte d'Ivoire, ou français local ivoirien (noté FI), le dioula de Côte d'Ivoire et le baoulé.

Le dioula (langue très proche du bambara) et le baoulé sont historiquement les véhiculaires ivoiriens ${ }^{4}$, que le français est venu rejoindre depuis un siècle dans ce rôle. Toutes ces langues et variétés font partie du paysage plurilingue dans lequel s'est créé et se développe le français en Côte d'Ivoire. Parmi ces langues, seul le baoulé n'est parlé qu'en Côte d'Ivoire, mais notre analyse concerne les faits linguistiques tels qu'ils se présentent en Côte d'Ivoire dans les trois langues. Les contacts linguistiques ont, en effet, généré une «influence réciproque » (Kouadio 2005 : 182) du français et des langues ivoiriennes, surtout celles qui ont une fonction véhiculaire.

D'un point de vue notionnel, nous faisons une large part à l'approche fonctionnelle de Denis Creissels, dont les concepts, élaborés dans une perspective typologique, ont l'avantage de cerner à la fois les faits du français standard, du français local ivoirien et des langues ivoiriennes.

Les constructions analysées appartiennent à la langue orale. Notre réflexion ne s'appuie pas uniquement sur des recensements à partir de corpus, mais aussi sur des connaissances complémentaires des langues en présence. Nous ne disposons donc pas de fréquence statistique pour les emplois nominaux des formes adpositionnelles étudiées; ils sont néanmoins bien représentés dans les discours. Les sources des énoncés rapportés sont variées : journaux ivoiriens, travaux de chercheurs (Creissels 1991 ; Creissels \& Kouadio 1977 ; Tymian et al. 2003), oral

\footnotetext{
${ }^{3}$ Mes observations rejoindront celles de Katja Ploog (2002 a, b) bien que je donnerai parfois d'autres analyses des faits observés.

${ }^{4}$ Les soixante à soixante-dix langues aujourd'hui parlées en Côte d'Ivoire sont issues de quatre grands groupes culturels et linguistiques qui dépassent les frontières du pays, ayant chacun une langue dominante (pour la Côte d'Ivoire) : groupes mandé (dioula), gur (sénoufo), kru (bété) et kwa (baoulé).
} 
transcrit à la volée. Les exemples construits qui les complètent font partie des énoncés les plus banals en Côte d'Ivoire.

\section{Fonctions nominales et adpositionnelles}

Le constituant nominal peut être défini, par référence à un nom prototypique, comme tout "segment de phrase qui occupe une position structurelle que pourrait occuper un nom propre» (Creissels 1991: 43). Les noms propres ont en eux-mêmes une valeur référentielle et peuvent figurer comme syntagmes nominaux. Au contraire, l'actualisation en discours du signifié virtuel d'un nom ordinaire passe par l'adjonction d'éléments le spécifiant et, en français standard, obligatoirement de déterminants. D'autres classes d'items sont auto-spécifiés et susceptibles d'avoir le rôle de constituants syntaxiques (sans adjonction de déterminant en français standard). Il s'agit, entre autres, des "pronoms» (Creissels 1991: 202-210), qui doivent avoir "le statut de constituants nominaux à part entière, et [...] pouvoir tirer directement leur référence de la situation d'énonciation » (Creissels 1991 : 204), comme en français lui, elle, ceci ou cela. Il s'agit aussi des « quasi-nominaux » (Voir 5).

Dans les phrases (1) à (6) du français local ivoirien, les segments PREP ( $/$ Pro) sont actualisés en discours et occupent des positions structurelles de nom dans la phrase : sujet (1), (3)-(6), complément direct (9), complément de préposition (2).

(9) Je n'achète pas pour lui (Je n'achète pas ce qu'il vend)

Un rappel théorique s'avère nécessaire pour bien poser le problème de ces énoncés. Les syntagmes adpositionnels seront analysés dans les trois langues, qui présentent des faits complémentaires pour l'élaboration d'une distinction entre nom et adposition.

\subsection{Noms vs. adpositions}

Le français distingue, à l'instar de nombreuses langues qui possèdent des adpositions, les syntagmes nominaux des syntagmes prépositionnels. Le syntagme nominal a une tete lexicale, le nom, qui peut avoir comme complément un syntagme prépositionnel. Dans le syntagme prépositionnel, la tête est la préposition, qui appelle un syntagme nominal complément ${ }^{5}$. Cette tête n'est pas référentielle et ne peut recevoir les marques flexionnelles et les spécifications d'un nom. Alors que les deux types de syntagmes peuvent se trouver en fonction de complément d'un verbe, seuls les syntagmes nominaux, référentiels, peuvent assumer les fonctions de sujet d'un verbe en français standard. Par ailleurs, ce sont les prépositions qui déterminent dans quelles positions syntaxiques les syntagmes dont elles sont têtes peuvent s'insérer.

De la même façon, le dioula distingue les syntagmes nominaux des syntagmes adpositionnels. L'ordre des constituants en dioula place le sujet en début de phrase et les têtes en fin de syntagmes [sujet - complément direct - verbe - complément postpositionnel]. Le syntagme postpositionnel [nom - postposition] est formé comme le syntagme complétif [nom complément - non tête], la postposition a la même position qu'un nom tête (Sangaré 1984 inter al.). En dioula, alors que certaines postpositions n'ont qu'une valeur abstraite, l'origine nominale d'autres adpositions reste très proche. Ces items assument, en plus de la fonction de préposition, des emplois référentiels,

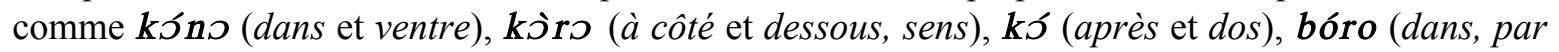

5 La préposition d'origine $d e$, toutefois, n'exclut pas un syntagme prépositionnel locatif (Voir plus loin l'exemple (46) et pour le français ivoirien Boutin (2005). 
et main). L'éloignement de la valeur référentielle n'est pas toujours nette mais une progression dans l'abstraction peut distinguer les divers emplois de bóro dans :

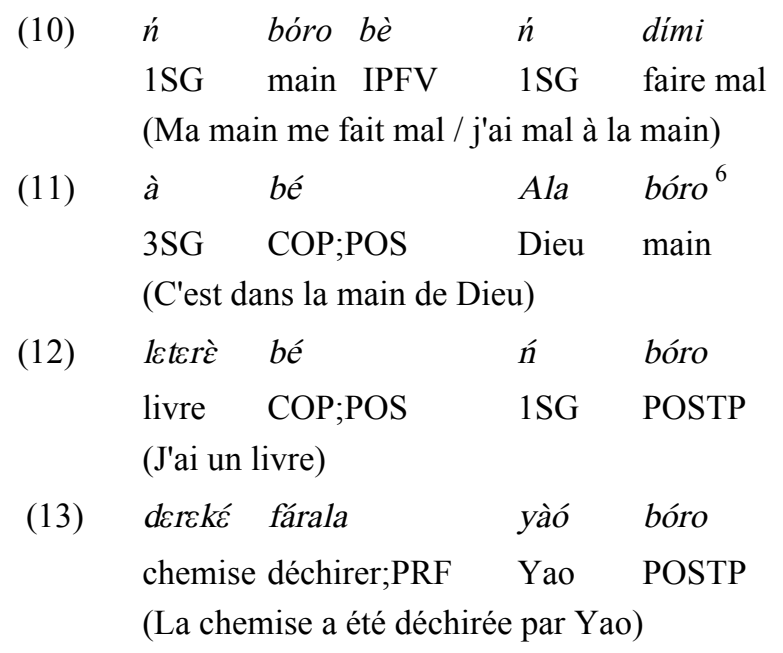

En (10) et (11), bóro est un nom (susceptible de recevoir des éléments tels que marques de pluriel, numéraux), tête de syntagme nominal. En (12) et (13), bóro est une postposition, tête d'un syntagme adpositionnel, obligatoire en (12) et facultatif en (13).

En baoulé, l'ordre des constituants est [sujet - verbe - premier complément - deuxième complément], mais dans les syntagmes complétifs, le complément du nom lui est antéposé. Les éléments qui introduisent un syntagme nominal pour former un syntagme de rang supérieur en fonction de circonstant ou de complément d'un nom ou d'un verbe ne constituent pas une classe d'adpositions (Creissels \& Kouadio 1977 : 347-354). S'ils sont d'origine nominale, ils conservent certaines de leurs propriétés, et le syntagme complétif formé est apte à occuper toutes les fonctions nominales dans la phrase. La langue utilise cependant, en fonction de postpositions locatives, des noms, par exemple de parties du corps, dont le sens peut évoquer une localisation plus générale (Creissels \& Kouadio 1977 : 354-365) : sú (sur et surface), nún (dans et intérieur), nyrún (devant et visage), sìn (derrière et dos), àfī̄n (au milieu de et taille), etc. Afī̄̄n nous aidera à montrer l'ambivalence de ces items '.

${ }^{6}$ L'énoncé à bé Ala bóro lá est possible aussi, lá étant une postposition locative qui met en évidence le caractère nominal de bóro. Charles Bailleul nous indique que la traduction bambara de Sag. 3,1 : la vie des justes est dans la main de Dieu, aucun tourment n'a de prise sur eux : mogo tilennenw ni be Ala bolo, tooro si te u soro bilen ne pose aucun problème en bambara sans la postposition (Kitabu Senu 2006).

${ }^{7}$ Les tons que je note sont «construits » selon le baoulé dit central (de Bouaké) par un locuteur fahri. Je les note ici bien que l'instabilité des tons baoulé ait amené les chercheurs à ne noter que certains tons grammaticaux lorsque l'absence de notation entrainerait des ambigüités (Voir Tymian et al. 2003). De fait, les tons du baoulé ne sont pas fixes pour tous les morphèmes, mais ils s'actualisent en discours et varient selon l'énonciation. Par ailleurs, le baoulé est constitué de nombreux dialectes qui se différencient notamment du point de vue tonal. 


$\begin{array}{lllll}\text { bian } & \text { ngá } & i & \text { àfiēn } & \text { cénnin } \\ \text { homme } & \text { DEM } & \text { 3SG } & \text { tronc } & \text { être grand }\end{array}$

(Cet homme a un gros tronc)

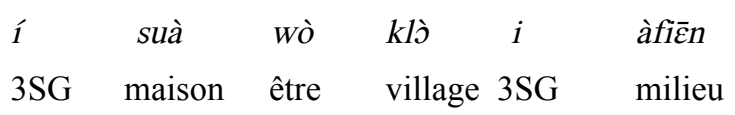

(Sa maison est au milieu du village)

(Creissels \& Kouadio 1977 : 361-362)

\begin{tabular}{|c|c|c|c|}
\hline (16) & 0 & wò & $b e$ \\
\hline & $3 \mathrm{SG}$ & être & $3 \mathrm{PL}$ \\
\hline
\end{tabular}

$B \boldsymbol{e}$ et $\boldsymbol{i}$ ne sont pas des déterminants, mais des pronoms de $3^{\mathrm{e}}$ personne, du pluriel et du singulier respectivement; antéposés à àfi $\bar{\varepsilon} n$, ils ont une fonction de complément de àfi $\bar{\varepsilon} n^{8}$, qui reste proche d'un nom même si sa valeur est plus abstraite dans (15) et (16). C'est ce qui permet au baoulé de positionner ce type de syntagme en fonction de sujet :

$\begin{array}{llll}\text { be } & \text { àfièn } & t i & \text { sàciwa } \\ \text { 3PL } & \text { entre } & \text { être } & \text { détérioré }\end{array}$

(Les relations entre eux sont rompues)

(Tymian et al. $2003: 60$ )

Judith Tymian et al. (2003) propose, dans un tel cas, une sémantisation de àfi $\bar{\varepsilon} n$ en « relation ». Le français ivoirien, de son côté, n'hésite pas à produire en parallèle de (17) la phrase : Entre eux est gâté.

Dans les énoncés dioula ou baoulé (10)-(17), la valeur référentielle du syntagme exclut son appartenance à la classe des syntagmes adpositionels. On a deux catégories de syntagmes : soit des syntagmes nominaux à valeur référentielle, soit des syntagmes adpositionnels à valeur non référentielle. Nous examinons à la suite la valeur référentielle qu'ont pourtant les formes adpositionnelles (1) à (8).

\subsection{Valeurs référentielles des formes PREP (N/Pro)}

Les énoncés (1) à (8) mettent en évidence le contexte anaphorique, propice à l'implicite, dans lequel les formes adpositionnelles étudiées sont le plus souvent utilisées. Cependant, dans (1) à (3) et (7)-(8), la relation entre l'antécédent et le référent implicite n'est pas une relation de coréférence. L'anaphore est de type uniquement lexical ou nominal (Kleiber 1994) : la seule identité entre l'antécédent et le référent implicite est une identité formelle. Dans ces phrases (1) à (3) les formes PREP (N/Pro) ont pour antécédent les noms têtes des syntagmes : du linge de ta voisine, couvrefeu de Gbagbo-là et le cours [de cuisine]. Dans (7), ń tá a pour antécédent móbili et dans (8), taluá lì̀ a pour antécédent tánní. Dans les phrases (4) à (6), nous avons aussi un contexte anaphorique puisque [le père] de la maman du locuteur, [le clignotant] de droite et [le clignotant] de gauche comme [les bananes] sont présents eux aussi dans la situation d'énonciation. L'antécédent

${ }^{8}$ Le pronom personnel antéposé à àfi $\mathbf{\varepsilon} \boldsymbol{n}$ en (14) et (15) est complément de àfi $\bar{\varepsilon} \boldsymbol{n}$. Le français n'a pas du tout le même ordre mais on peut aider à la représentation de ces phrases de la façon suivante : Sa maison est au village milieu de lui et Cet homme tronc de lui est gros. 
est absent du discours antérieur mais déjà présent et saillant dans le paysage mental des interlocuteurs (voir Kleiber 1994).

En outre, dans les exemples en français (1) à (6), les syntagmes nominaux à tête implicite ne sont pas issus de la réduction d'un syntagme nominal complet les précédant dans l'énoncé. Ils sont construits directement à partir de la représentation mentale, par anaphore vide, du référent. C'est de la même façon qu'on peut écrire sur une boîte :

A Jean / De Paris. / Pour les maux de tête

Ces titres ou étiquettes, cependant, ne peuvent normalement pas figurer dans une phrase en tant que constituant nominal en français standard:

*J'ai rangé à Jean / *Je n'ai pas vu de Paris / *J'ai pris pour les maux de tête.

Dans les énoncés étudiés, l'absence d'un référent explicite entraîne la mise en place de fonctions pragmatiques. D'un point de vue cognitif, la référence est possible par un processus métonymique :

La métonymie est un processus cognitif par lequel une entité conceptuelle fournit un accès mental à une autre entité conceptuelle. Le principe en oeuvre est celui de la contiguïté [souligné par l'auteur] où un rapport est établi entre deux entités à l'intérieur de ce domaine. La nature de la contiguité est conceptuelle, car la métonymie fait appel à une représentation mentale où le sujet mobilise ses connaissances extra-linguistiques. Si celles-ci font défaut, le mécanisme référentiel ne joue pas (Fougner Rydning 2003).

Dans les cas qui nous occupent, les noms explicites sont effectivement introduits par des prépositions spécialement aptes à établir des relations de contiguïté. Nous avons des prépositions directement locatives dans les phrases (5) et (6). Dans la phrase (4), il s'agit d'un repérage familial, dans la phrase (3), d'un repérage temporel. Les phrases (1)-(3) et (7)-(8) présentent des syntagmes indiquant l' «appartenance », dans le sens d' "être propre à » ${ }^{9}$. D'un point de vue sémantique, l'« appartenance » est un cas de localisation, exprimée dans de nombreuses langues par une prédication de localisation. C'est, par exemple, le cas du dioula (Voir (12)). Dans toutes ces phrases, l' « accès mental » au référent implicite a été permis par l'adposition (français et dioula) ou le nom tête lì̀ (baoulé), et le syntagme adpositionnel explicite, qui l'ont spécifié par sa situation, physique ou abstraite.

A part dans (8) où lì̀ est un nom, le statut de constituant nominal des syntagmes adpositionnels (1)-(7) est associé à une tête vide. La comparaison avec d'autres syntagmes nominaux à tête vide dans les trois langues nous aidera à apprécier, d'une part, la capacité du français à recevoir de telles structures et, d'autre part, le partage de procédures communes chez les locuteurs des trois langues.

\section{Les syntagmes nominaux à tête vide}

En français standard, mis à part le cas des syntagmes prépositionnels titres et étiquettes du type des exemples (18) qui ne peuvent être constituants nominaux dans une phrase, des syntagmes nominaux à tête vide sont possibles comme constituants syntaxiques. Ils sont issus de réductions de syntagmes nominaux pleinement explicites par l'ellipse du nom tête, telles que : La voiture de Guy et celle d'Eve.

${ }^{9}$ Le concept de « possession » est, quant à lui, discutable. Je reviendrai plus en détail sur la valeur sémantique de pour dans la section 4. 
L'analyse en termes de réduction est celle que Creissels (1991: 57-62) propose en particulier pour le français et le bambara ${ }^{10}$, qui fonctionnent de façon similaire sur ce point. Dans ces deux langues, la syntaxe permet l'ellipse du nom tête anaphorique; celui-ci reste récupérable par la conservation des éléments qui lui sont normalement associés : en français, le déterminant et le syntagme prépositionnel complément, et en dioula, le syntagme postpositionnel complément seulement.

\subsection{En français standard}

On a en français standard, pour des syntagmes nominaux formés d'un nom tête et d'un modifieur adjectival ou prépositionnel, les réductions suivantes :

$$
\begin{aligned}
& \text { Il a une voiture verte et sa femme une grise } \\
& \text { Ce chien-là est méchant, pas celui-ci } \\
& \text { J'ai pris le sac de Guy et celui d'Eve } \\
& \text { Il n'a pas vu le sac de Guy, il a vu le mien }
\end{aligned}
$$

Dans toutes ces phrases, le deuxième syntagme nominal est réduit et n'est constitué que du déterminant et d'un adjectif ou d'un complément du nom. Le nom tête, quant à lui, est implicite. En (22) la réduction s'accompagne de l'apparition d'un morphème spécialisé mien en fonction d'indice génitival $^{11}$.

Le déterminant reste inchangé dans (19) et dans (22). Dans le cas particulier des syntagmes génitivaux, le français a recours à celui et son paradigme (celle, ceux), comme en (21), que Creissels (1991: 201-210) n'analyse pas comme des pronoms, mais comme des variantes combinatoires du déterminant dans les syntagmes complétifs réduits. En effet, celui-ci a le même rôle que ce chien-ci et n'est pas pronom comme le sont ceci ou cela ${ }^{12}$. En outre, celui, une, ce et le ont la même distribution dans les syntagmes réduits et non réduits de (19) à (22). Par ailleurs, dans le cas d'un syntagme génitif, la préposition de ne change pas dans la construction réduite de (21), contrairement à ce qui se passe en français ivoirien dans (1) et (2) (Voir aussi 4).

La réduction de syntagmes nominaux en contexte d'anaphore par l'ellipse de la tête se retrouve aussi en dioula, avec les modalités qui lui sont propres.

${ }^{10}$ Voir Aby Sangaré (1984 : 280-289) pour le dioula sur ce point.

${ }^{11}$ Dans des états antérieurs de la langue ou dans d'autres langues romanes, des syntagmes génitifs sont encore possibles sous cette forme. Nathalie Fournier (1998: 141) répertorie quelques exemples en français classique, du type : Il m'est mort un mien frère (La Fontaine). Nous avons, en italien : Il nostro libro, en espagnol : El amigo mío.

${ }^{12}$ En français, ceci, cela sont de « véritables pronoms » (Creissels $\left.1991: 202-210\right)$. «L'analyse en termes de réduction de syntagme ne leur est pas applicable : ils s'emploient pour référer sans les identifier au signifié d'un lexème substantival à des choses présentes dans la situation d'énonciation: de même que lui ou elle est le signe vocal qui accompagne le geste désignant une personne présente dans la situation, de même ceci ou cela accompagnent le geste qui désigne une chose » (Creissels 1991 : 203). D'autres pronoms, comme autrui analysé par Catherine Schnedecker (1999), ont des propriétés et des mécanismes référentiels particuliers. Celui, pour sa part, semble ne pas pouvoir être assimilé à un pronom. 


\subsection{En dioula}

En dioula ${ }^{13}$, les déterminants et modifieurs sont postposés au nom tête, comme le démonstratif nìn dans wùlú nìn (ce chien) dans (23) et l'adjectif blémán dans mìsi blémán (vache rousse) dans (24).

$\begin{array}{lllll}\text { (23a) wùlú nìn } & \text { ká } & \text { júgú } \\ & \text { chien } & \text { DEM } & \text { COP;POS } & \text { méchant } \\ \text { (Ce chien est méchant) } & \\ \text { (24a) } & \grave{a} & \text { kà } & \text { mìsi } & \text { blémán sán } \\ & \text { 3SG } & \text { PRF } & \text { vache } & \text { rousse acheter }\end{array}$

(Il a acheté une vache rousse) (Creissels 1991:59)

Le syntagme complétif est formé du nom complément, antéposé, et du nom qu'il complète. Le syntagme génitif est construit, dans sa forme la plus longue, du nom « humain » ou de l'affixe de personne, de la postposition tá, le tout antéposé au nom tête, comme à tá móbili (sa voiture) dans (7).

\begin{tabular}{llccc}
$\grave{a}$ & mà & \multicolumn{1}{c}{} & tá & móbili bòri \\
3SG & PRF-NEG & 3SG & POSTP & voiture conduire \\
(Il n'a pas conduit sa voiture) &
\end{tabular}

Cette forme avec tá est généralement utilisée pour des noms têtes «aliénables », alors que la forme des groupes génitifs « inaliénables » se caractérise par l'absence de la postposition tá et la juxtaposition du complément au nom-tête (Voir (25)). Les divers facteurs d'apparition de tá sont étudiés par Sangaré (1984) sur la base de critères sémantiques (relations naturelles ou acquisition, émanations, relations parentales, de réciprocité, hiérarchiques, etc.). La notion d' "aliénabilité/inaliénabilité » en linguistique est précisée dans Creissels (2001), qui montre l'impossibilité d'opérer une distinction sémantique générale entre les deux types de relations.

Des syntagmes nominaux à tête vide peuvent apparaître en contexte anaphorique en dioula (comme en français standard), par exemple dans le cas d'une détermination démonstrative :
wùlú nìn ká júgú,
nìn mán júgú
chien DEM COP;POS méchant,
DEM COP;NEG méchant

(Ce chien est méchant, pas celui-ci)

dans le cas de modifieurs adjectivaux :

$\begin{array}{llllllll}\text { (24b) à } & \text { kà } & \text { mìsi blémán } & \text { sán, ní finmán } \\ & \text { 3SG } & \text { PRF;POS } & \text { vache } & \text { rousse } & \text { acheter et } & \text { noire }\end{array}$

(Il a acheté une vache rousse et une noire) (Voir Creissels $1991: 59$ )

${ }^{13}$ La structure du groupe nominal est étudiée, pour le dioula, par Dumestre \& Retord (1981); Sangaré (1984 : 280-289), auxquels nous empruntons la plus grande partie des analyses qui suivent. Les exemples (23) et (24) sont repris des exemples en bambara de Creissels (1991), adaptés au dioula. 
ou encore dans le cas d'un syntagme génitif (réduit), qui nous intéresse ici :

\begin{tabular}{|c|c|c|c|c|}
\hline à & mà & $\grave{a}$ & tá & móbili bòri, \\
\hline $3 \mathrm{SG}$ & PRF-Nég & $3 \mathrm{SG}$ & POSTP & voiture conduire, \\
\hline ̀̀ & kà & ń & tá & bòri \\
\hline SG & PRF;POS & $1 \mathrm{SG}$ & POSTP & conduire \\
\hline
\end{tabular}

Dans le syntagme génitif à nom tête inaliénable, tá n'apparaît pas, sauf en cas de réduction par l'ellipse du nom tête :

$\begin{array}{rllllll}\text { (25a) dioula : } & \grave{a} & \text { dén } & \text { lò } & \text { / à } & \text { tá } & \text { lò } \\ & \text { 3SG enfant } & \text { PRES } & \text { / 3SG } & \text { POSTP PRES } \\ & \text { (C'est son enfant } & \text { / C'est le sien) }\end{array}$

Comme on le voit en (7) et (25a), en cas d'anaphore et de réduction du syntagme génitif par l'ellipse du nom tête, tá occupe sa fonction de postposition. En effet, en dioula véhiculaire, le syntagme réduit ( $N$ / Pro) tá ne reçoit pas l'adjonction des marques «défini », "indéfini », «pluriel » qui caractérisent les noms. Cela confirme que tá n'a pas un statut syntaxique de nom mais est bien une postposition. On a, par exemple :

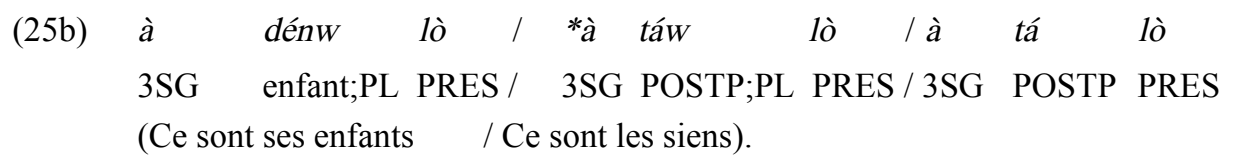

Dans (7) et (25), tá est donc bien une postposition, tête du syntagme postpositionnel, et il ne constitue pas un nom. Le nom tête est implicite, une anaphore vide, comme dans (1)-(6). Le syntagme postpositionnel peut alors continuer d'être interprété comme spécifiant un terme structurellement présent mais non explicité, dont le contexte permet de rétablir l'identité.

Il est possible que la structure tá ( $N$ / Pro) offre un schéma pour l'utilisation de pour ( $N$ / Pro) dans (1)-(3). Cependant, on ne peut encore déterminer dans quelle mesure le français (ivoirien) n'aurait pas aussi facilité le transfert de tá de la catégorie des noms à celle des adpositions. En effet, contrairement au dioula, le bambara (Mali), distingue deux formes: la postposition ká du complément du syntagme génitif non réduit, et le nom tá (de sens « chose », " part ») lorsque celuici est réduit. En bambara, tá peut recevoir les morphèmes de spécification qui se placent normalement à la fin du syntagme nominal : à tá-w lò (3SG POSTP-PL PRES) (Ce sont les siens). Le dioula représente un état de langue où la valeur nominale de ce morphème s'est perdue, du fait de son utilisation comme postposition.

En tout état de cause, la possibilité que la préposition elle-même des formes PREP (N / Pro) ait un statut nominal doit aussi être examinée aussi. En effet, le français en Côte d'Ivoire est en contact avec des langues, telles que le baoulé, dans lesquelles le rôle des adpositions est assuré par des items d'origine nominale (Voir 2.1). 


\subsection{En baoulé}

Après un bref rappel de la structure des syntagmes génitifs en baoulé, nous comparons ici pour ( $N /$ Pro) en français local ivoirien et ( $N /$ Pro) lì̀ en baoulé. Ils sont sémantiquement équivalents mais les propriétés syntaxiques de pour et de lič sont cependant distinctes.

Dans les syntagmes génitifs, le nom complément est antéposé au nom tête ; un pronom de rappel peut éventuellement lui être antéposé aussi, comme en (26). Chacun des deux noms peut recevoir des marques de spécification, « défini », « indéfini », « pluriel », etc. :

\begin{tabular}{llll} 
táluà'n & \multicolumn{1}{l}{ tánnín’n } & ò \\
fille;DEF & 3SG & pagne;DEF & PRES \\
(C'est le pagne de la fille)
\end{tabular}

On ne peut pas parler, en baoulé, de réduction du syntagme génitif, mais d'une substitution grâce

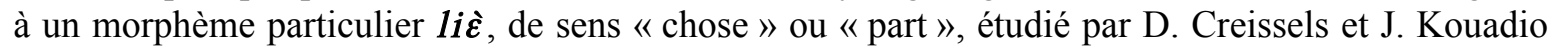
(1977 : 327-341). Li⿳亠㐅⿵ (nic̀ après un mot à finale nasale) est un nom à complément obligatoire qui peut se substituer au nom tête d'un syntagme génitif en contexte anaphorique, pour constituer un syntagme de structure $(N+$ Pro) lic. Ainsi :

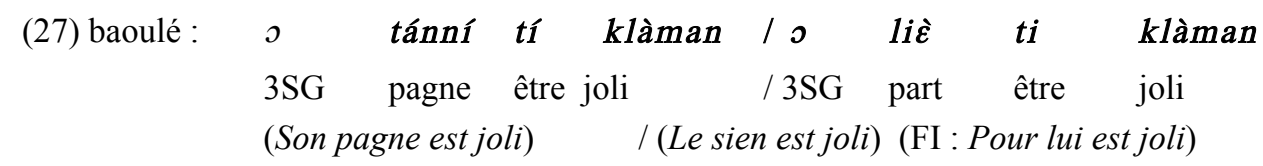

Liغ̀ n'a pas perdu sa nature nominale et peut donc porter toutes les marques de spécification du nom :

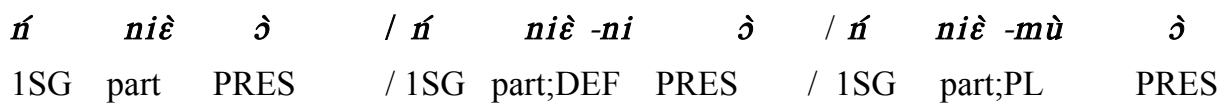

$$
\begin{aligned}
& \text { (C'est à moi. /C'est le mien. / Ce sont les miens.) }
\end{aligned}
$$

Cette dernière propriété différencie liè du baoulé de tá du dioula et de pour du français ivoirien, pour lesquels aucune spécification n'est possible. tá et pour sont bien une postposition et une préposition respectivement, contrairement au nom liø̀ du baoulé. Néanmoins, tá et pour n'ont pas les mêmes propriétés: alors que tá construit des syntagmes complétifs, pour n'en a pas la possibilité.

\section{Les prépositions des formes PREP (N / Pro) du français ivoirien}

Pour, qui entre très facilement dans une forme $\operatorname{PREP}(N /$ Pro), ne peut régir le complément d'un nom explicite en français ivoirien actuel. Ce fait nous amène à tester les propriétés des prépositions susceptibles d'entrer dans une forme PREP ( $N$ / Pro), en contraste avec celles des prépositions normalement utilisées pour introduire des syntagmes complétifs, telles que de et $\grave{a}$.

Les prépositions de et $\grave{a}$ des syntagmes génitifs ne peuvent entrer dans une structure de forme PREP (N / Pro) qui occupe une fonction nominale. On a ainsi, en contraste avec les phrases (4) et (5) : 
(4a) Le père (de / à) ton papa est toujours là ?

- *Non, c'est (de / à) ma maman qui est encore là.

(5a) Est-ce que le clignotant de droite marche?

- *De gauche marche mais de droite ne marche pas.

Dans les énoncés (1) et (2), il est impossible de remplacer pour par de ou à, tout comme il est impossible de remplacer de ou à par pour :

(1a) *Avant de te moquer du linge pour ta voisine, regarde si pour toi est propre.

(2a) *Couvre-feu de Gbagbo-là, ce n'est pas comme de Guéïlà !

Par ailleurs, à l'instar de pour, les prépositions locatives chez, dans, à, ne peuvent pas toujours introduire un complément du nom :

(4b) * Le (père /grand-père) chez ton papa est là ?

(5b) ? Le clignotant à droite marche.

(6a) Les bananes (qui sont) dans la cour sont mûres.

Ces propriétés syntaxiques différencient les prépositions locatives (au sens large) chez, dans, à, pour, des prépositions génitives de et $\grave{a}$.

La valeur locative de chez, dans, à et pour dans les exemples (1) à (6) a déjà été montrée en 2.1 . La valeur d' « appartenance » de pour peut être dérivée de celle de " qui concerne ». Cette valeur est celle de certains de ses emplois en français standard, où il peut introduire un nom comme le fait quant à $N$, en ce qui concerne $N$ dans des phrases comme :

Pour la facture, je la règlerai demain.

Pour nous, nous n'avons eu que peu de dégâts.

Lorsque pour a la valeur « concernant $N$ », il est apte à opérer un repérage mental, situer un cadre. D'un point de vue syntaxique, ces emplois sont tout à fait différents de ceux des formes pour ( $N$ / Pro) puisque, dans (29) et (30), les séquences pour ( $N$ / Pro) constituent des compléments de phrase et ne peuvent avoir une fonction actancielle dans la phrase. C'est pourtant avec cette valeur sémantique de « qui concerne » que pour est utilisé dans les formes pour ( $/$ Pro) du français local ivoirien, comme une voie d'accès au concept d'appartenance à la sphère personnelle (Creissels, $1979,2001)^{14}$, opérant ainsi une localisation.

Il peut être intéressant de noter ici que ( $N /$ Pro) lì̀ est fréquemment utilisé, cette fois sans marque de flexion nominale, pour thématiser un nom comme dans :

${ }^{14}$ Creissels 1979, dans l'optique typologique de sa thèse d'état, conteste la notion de «possession ». Il lui préfère celle de « participation à la sphère personnelle ». La notion de «sphère personnelle » découle du fait que l'homme a conscience d'un réseau de relations qui l'entourent, par lesquelles il se sent plus ou moins concerné. La « possession », autrement dit le fait d'exercer un droit de regard sur certains objets, n'est qu'un cas particulier. Le concept de "possession» peut alors être restrictif, tout comme la dénomination correspondante «possessif» peut être impropre, chaque fois que la construction ne réfère pas au droit social ainsi désigné. Dans la plupart des cas, la spécification d'un nom par un complément « humain » n'est qu'une association logique d'une entité à une autre entité, humaine. Creissels 1979 propose le terme général de valeur «associative» pour le rattachement à la sphère personnelle. Par la suite il utilise celui de « génitif » dans le même sens (Creissels 1991). 
yàci, wò liè, a kwlá wànndi tra mìn

pardon, 2SG quant à 2SG pouvoir courir dépasser $1 \mathrm{SG}$

(F : S'il te plait, toi, tu peux courir plus vite que moi) (Tymian)

On peut comparer ses deux emplois dans :

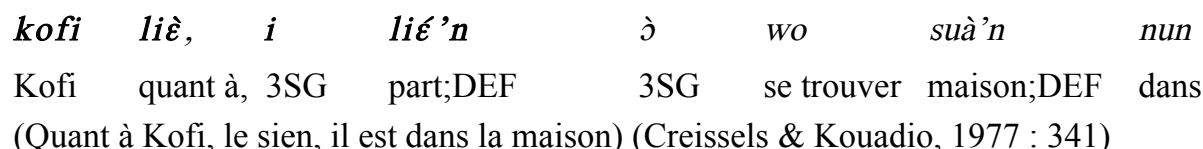

Liè peut donc perdre ses propriétés nominales lorsqu'il construit un syntagme détaché, de sens « quant à $(N /$ Pro $)$ ».

Pour conclure, en termes de catégorisation, premièrement les formes PREP ( $/$ Pro), y compris celles en pour ( $N /$ Pro), ne sont pas des syntagmes nominaux complétifs, deuxièmement, les prépositions des formes PREP ( $N /$ Pro) sont des prépositions qui opèrent une localisation soit physique soit plus ou moins abstraite.

Nous sommes donc en présence de syntagmes adpositionnels locatifs (ou de repérage) de type adverbial, ce qui nous autorise à comparer leur syntaxe à celle de certains adverbiaux qui eux aussi assument des fonctions nominales.

\section{Propriétés nominales de certains éléments adverbiaux}

Notre but est ici d'évaluer la résistance des quatre langues ou variétés à des emplois nominaux des formes adpositionnelles en question. De fait, on observe en français local ivoirien, mais aussi en français standard et dans les langues ivoiriennes, des cas de fonctions nominales occupées par des adverbiaux (ou circonstants). Le statut particulier de ces adverbiaux conduit Creissels (1991) à poser l'existence d'une sous-classe intermédiaire de « quasi-nominaux ». Cette analyse éclaire aussi le fonctionnement des formes PREP (N/Pro), bien que les procédés référentiels associés aux formes PREP (N/Pro) et aux éléments adverbiaux utilisés comme noms demeurent différents.

\subsection{En français local ivoirien}

Dans notre variété, des éléments adverbiaux sont aptes à occuper des fonctions nominales, qu'ils aient une structure morphologique adverbiale, comme demain, maintenant, ici, là-bas... ou qu'ils aient la forme d'un syntagme prépositionnel. Les exemples sont :

Pour ton problème, il faut demain (Pour ton problème, on verra demain)

Demain trouvera Yao au marché (Yao sera encore au marché demain)

En même temps est mieux (Le moment présent est le meilleur pour agir)

Maintenant est mieux (Maintenant c'est mieux)

Comme ça n'est pas bon (Cette façon de faire n'est pas la bonne)

Ici est bien (Cet endroit est bien.)

J'ai essayé de le joindre, mais chez lui ne répond pas (oral 02)

Dans ces phrases, les éléments adverbiaux appartiennent à la classe d'éléments que Creissels (1991: 207-209) appelle « quasi-nominaux », qui «ont le statut de constituants nominaux à part entière, et [...] la particularité de pouvoir tirer directement leur référence de la situation d'énonciation. »(Creissels 1991 : 208). 
Les adverbiaux « quasi-nominaux » assument des fonctions de sujets dans les exemples (34) à (39) et une fonction de complément dans l'exemple (33). Une identité de forme apparaît entre ces phrases et les phrases (1) à (6). Cependant, une différence fondamentale d'interprétation existe entre ces deux types de phrases, que l'on peut mettre en évidence par la comparaison de (6) et (38), puis de (4) et (39). Dans (38), ici a les propriétés sémantiques et syntaxiques de cet endroit. Dans (6), au contraire, ce n'est pas (ici / cet endroit) qui est mûr mais les bananes qui sont ici. Ici réfère aux bananes par métonymie (Voir 2.1). De la même façon, dans (39) chez lui est substituable par son domicile, alors que dans (4) chez ma maman n'a pas pour référent le domicile de ma maman, mais le père de la maman.

Malgré cette disparité fonctionnelle, les énoncés (33) à (39) montrent que le français ivoirien possède un cadre formel pour que des syntagmes prépositionnels assument des fonctions essentielles dans une phrase.

\subsection{En français standard}

Les éléments que nous avons appelés « quasi-nominaux » pour (33) à (39) vont être perçus, en français standard, comme des adverbes ou circonstants et ne pourront pas, généralement, assumer des fonctions nominales. La fonction de sujet, en particulier, est souvent impossible : le clitique ce est alors obligatoire, référant à une situation ou un événement appréhendé par les interlocuteurs et pourvu d'une capacité de référence directe à cette situation ou à cet événement :

En même temps c'est mieux / Maintenant c'est mieux / Comme ça ce n'est pas bon

De même, dans les exemples (5) et (6), un pronom ou clitique s'impose comme élément référentiel en français standard:

(41) [Les clignotants] A droite (il / ça) s'allume mais à gauche (il / ça) reste éteint

(42) [Les bananes] Ici elles sont mûres, dans la cour elles ne sont pas mûres

De tels adverbes et syntagmes prépositionnels peuvent pourtant occuper dans certains cas des fonctions syntaxiques nominales en français : complément verbal (43), sujet (44), complément de nom (45), complément de préposition (46).

(43) Préfères-tu maintenant ou demain ? / Préfères-tu à droite ou à gauche ? /

Préfères-tu le vélo ou la voiture?

(44) Maintenant m'irait mieux. / A gauche m'irait mieux. / La voiture m'irait mieux

(45) Les gens d'ici / Les gens comme ça / Les achats d'hier / Les gens de la ville / Les achats de Guy

(46) Il vient de (derrière la maison / chez lui / dans la maison) / Il vient de la maison

Comme le montrent ces phrases, l'adverbe peut commuter avec un syntagme nominal. Cette propriété fait clairement apparaître qu'un statut nominal est possible pour ces éléments adverbiaux en français standard. Dans une autre optique, nos analyses rejoignent celles de C. Fabre et D. Bourigault (2008), qui montrent qu'il existe un continuum entre arguments et circonstants.

Les cas (43) à (46) demeurent cependant restreints et les énoncés (33) ou (35) sont à la limite de l'intercompréhension pour des locuteurs non ivoiriens. Les procédures interprétatives sont mieux partagées avec des locuteurs dioula ou baoulé. 


\subsection{Dans les langues ivoiriennes}

En baoulé et en dioula, il existe une grande facilité pour que des éléments adverbiaux occupent des positions nominales et s'insèrent donc dans la classe des « quasi-nominaux » (Creissels 1991).

Le dioula possède les énoncés suivants, proches des exemples (34) à (39), où des adverbes occupent des fonctions de sujet. Dans chacun de ces exemples, l'adverbe déictique peut commuter avec un syntagme nominal :

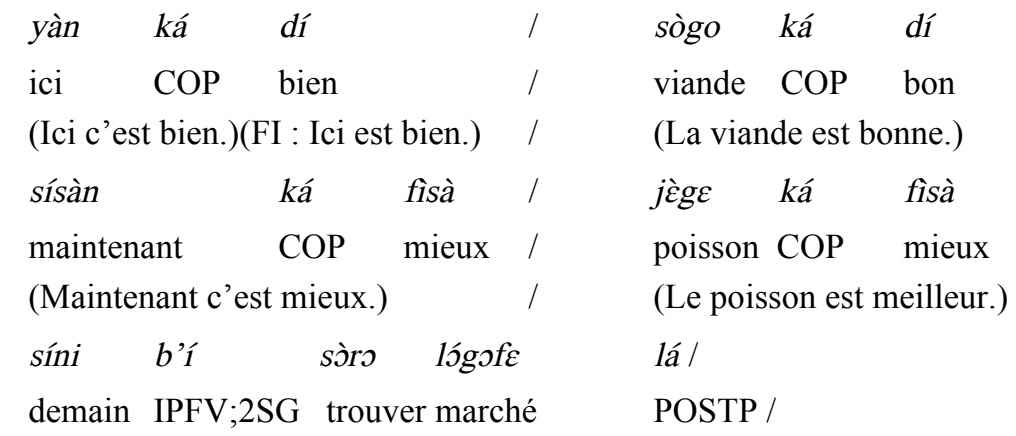

(Demain on te trouvera au marché.) (FI : Demain te trouvera au marché.) /

$\begin{array}{lllll}\text { Awa } & \text { b’í lòro lógofe } & \text { lá } \\ \text { Awa } & \text { IPFV;2SG trouver } & \text { marché } & \text { POSTP }\end{array}$

(Awa te trouvera au marché.) (Voir Creissels 1991 : 208)

Dans ces phrases, yàn, sísàn et síni ont un statut de constituant nominal, comme les noms sògo, jहg $\varepsilon$ et $A w a$, et une référence directe dans la situation d'énonciation. Ils n'ont pas de fonction adverbiale.

Le baoulé manifeste la même capacité, dans des phrases équivalentes :

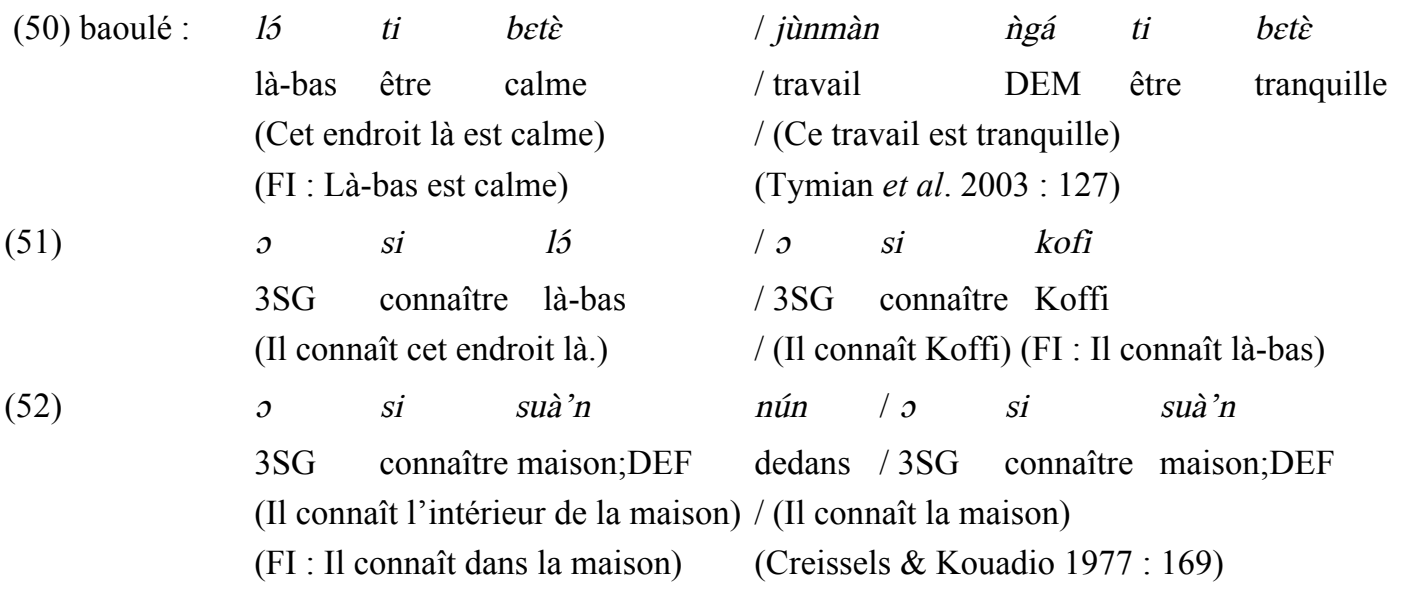

Dans la phrase (50), 15 (là-bas) a une fonction de sujet comme jùnmàn rìgá (cet homme) ; dans (51) et (52), 15 (là-bas) et suà'n nún (dans la maison), comme Kofi et suà'n (maison), ont une fonction de complément.

Toutes ces phrases ont des équivalents en français local ivoirien, comme nous l'avons indiqué entre parenthèses et on observe dans les trois langues de Côte d'Ivoire une grande accessibilité des 
adverbiaux à des fonctions nominales. Ces langues se distinguent par là du français standard, qui fournit une certaine résistance à employer des formes adverbiales en fonction de noms.

\section{Conclusion}

Après l'examen minutieux de l'hypothèse d'un transfert des langues ivoiriennes vers le français, notre étude a démontré sur un point précis l'impossibilité d'une explication simpliste des particularités morphosyntaxiques du français d'Abidjan comme résultat de transferts à partir de langues africaines en contact.

Certaines convergences sont indiscutables. Tout d'abord, dans les trois langues en contact, une démarcation nette entre adverbiaux et nominaux est remise en cause par la double possibilité des locatifs, et adverbiaux en général, d'être arguments ou circonstants. Par ailleurs, certains syntagmes adsitionnels, en localisant un référent par un processus de métonymie, acquièrent la propriété d'assumer formellement des fonctions nominales. Enfin, les trois langues possèdent les formes pour

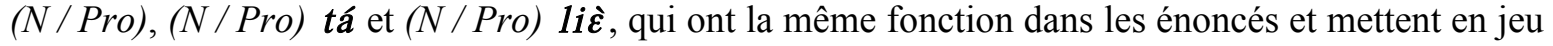
des habitudes pragmatiques communes.

Cependant, d'une part, la fonction d'adposition est diversement assumée dans les trois langues, qui ne permettent pas de réelles équivalences syntaxiques, d'autre part, les formes pour ( $N$ / Pro), ( $N$ / Pro) tá et ( $/$ Pro) liè présentent des contrastes structurels irréductibles.

\section{Notations :}

Les notations ne correspondent que partiellement aux 'Leipzig Glossing Rules' du Max-PlanckInstitut.

$\begin{array}{ll}\text { PRF, IPFV } & \text { correspondent respectivement aux valeurs « accompli » et « inaccompli » } \\ \text { COP } & \text { correspond à une copule } \\ \text { 1/2/3, SG/PL } & \text { correspondent aux } 1^{\mathrm{e}}, 2^{\mathrm{e}} \text { et } 3^{\mathrm{e}} \text { personnes du singulier et du pluriel } \\ \text { POS, NEG } & \begin{array}{l}\text { correspondent respectivement aux valeurs « positif » et « négatif » associées } \\ \text { à des morphèmes verbaux }\end{array} \\ \text { POSTP } & \text { correspond à une postposition associée à un nom } \\ \text { PRES } & \text { correspond à un présentatif } \\ \text { Pro } & \text { correspond à un pronom } \\ \text { N } & \text { correspond à un constituant nominal } \\ \text { DEF, DEM } & \text { correspondent aux morphèmes « défini » et « démonstratif » associés à des } \\ & \text { noms. }\end{array}$

Les langues ivoiriennes apparaissent avec l'orthographe officielle pour l'Afrique de l'Ouest, qui ne correspond pas totalement à l'API (alphabet phonétique international). Comme il est d'usage lorsque les tons sont notés, seul les tons principaux haut et bas le sont, les tons secondaires et moyens pouvant être déduits (Voir aussi la note 7). 


\section{Références}

Boutin, Béatrice Akissi. 2002. Description de la variation: Etudes transformationnelles des phrases du français de Côte d'Ivoire. Villeneuve sur Ascq: Presses Universitaires du Septentrion.

Boutin, Béatrice Akissi. 2005. L'alternance de prépositions dans les constructions locatives en français en Côte d'Ivoire à la lumière du dioula et du baoulé. Cahiers de l'Institut de Linguistique de Louvain 31-1 : 5-34.

Boutin, Béatrice Akissi. 2006. Extension de pour "possessif» dans le lexique français de Côte d'Ivoire et contacts de langues. Le français en Afrique 21 : 291-302.

Creissels, Denis. 1979. Les constructions dites «possessives », étude de linguistique générale et de typologie linguistique. Thèse d'état. Université Paris IV.

Creissels, Denis. 1991. Description des langues négro-africaines et théorie syntaxique. Grenoble : Éditions littéraires et linguistiques de l'Université de Grenoble (Ellug).

Creissels, Denis. 2001. Catégorisation et grammaticalisation. La relation génitivale en mandingue. In : Robert Nicolaï (éd.), 433-454. Leçons d'Afrique. Filiations, ruptures, et reconstitution de langues. Hommage à Gabriel Manessy. Louvain - Paris : Peeters.

Creissels, Denis \& N'Guessan Jérémie Kouadio. 1977. Description phonologique et grammaticale d'un parler baoulé. Abidjan : Institut de Linguistique Appliquée.

Dumestre, Gérard \& Georges L.A. Retord. 1981. Kó dì ? Cours de dioula. Abidjan, Dakar, Lomé : Nouvelles Editions Africaines.

Fabre, Cécile \& Didier Bourigault. 2008. Exploiter des corpus annotés syntaxiquement pour observer le continuum entre arguments et circonstants. Journal of French Language Studies $18-1: 87-102$.

Fougner Rydning Antin. 2003. La métonymie conceptuelle. In : Kåre Nilsson, Solveig Schult Ulriksen \& Hallvard Dørum (eds.), Romansk Forum 17 : 71-84. Oslo: Universitetet i Oslo. http://www.duo.uio.no/roman/Art/Rf17-03-1/06.Rydning.pdf

Fournier, Nathalie. 1998. Grammaire du français classique. Paris : Belin.

Kitabu Senu Layidukoro Sebenw. 2006. (Ancien Testament) trad. Charles Bailleul \& Vittorio Bonfanti. Bamako : Editions Donniya.

Kleiber, Georges. 1994. Anaphores et pronoms. Louvain-la-Neuve : Duculot.

Kouadio, N'Guessan Jérémie. 2005. Le nouchi et les rapports dioula / français. Hommage à Suzanne Lafage. Le français en Afrique 21 : 177-191.

Leipzig Glossing Rules. Conventions for interlinear morpheme-by-morpheme glosses. Max Planck Institute for Evolutionary Anthropology. http://www.eva.mpg.de/lingua/resources/glossingrules.php

Ploog, Katja. 2002a. Le français à Abidjan. Pour l'approche syntaxique du non-standard. Paris : CNRS-Éditions.

Ploog, Katja 2002b. Une promotion pour les locatifs en abidjanais ? Revue Romane 37-1 : 31-43.

Sangaré, Aby. 1984. Dioula de Kong (Côte d'Ivoire) : phonologie, grammaire, lexique et textes. Thèse de 3e cycle. Université Grenoble 3. 
Schnedecker, Catherine. 1999. Autrui : un pronom vraiment pas comme les autres. Journal of French Language Studies 9-1 : 69-90.

Tymian, Judith, N'Guessan Jérémie Kouadio, Jean-Noël Loucou. 2003. Dictionnaire baoulé français. Abidjan : Nouvelles Editions Ivoiriennes.

Weinreich, Uriel. [1953] 1967. Languages in contact. Findings and Problems, The Hague: Mouton $\&$ Co. 